\title{
Safe Implementation of Computerized Provider Order Entry for Adult Oncology
}

D.B. Martin'; D. Kaemingk'; D. Frieze ${ }^{2}$; P. Hendrie'; T.H. Payne ${ }^{3}$

${ }^{1}$ Seattle Cancer Care Alliance, Seattle, WA, United States; ${ }^{2}$ Department of Pharmacy, University of Washington Medical Center/ Seattle Cancer Care Alliance, Seattle, WA, United States; ${ }^{3}$ Departments of Medicine, Health Services and Biomedical \& Health Informatics, University of Washington, Seattle, WA, United States

\author{
Keywords \\ CPOE, chemotherapy, patient safety, transplant
}

\section{Summary}

Background: Oncology has lagged in CPOE adoption due to the narrow therapeutic index of chemotherapy drugs, individualized dosing based on weight and height, regimen complexity, and workflows that include hard stops where safety checks are performed and documented.

Objectives: We sought to establish CPOE for chemotherapy ordering and administration in an academic teaching institution using a commercially available CPOE system.

Methods: A commercially available CPOE system was implemented throughout the hospital. A multidisciplinary team identified key safety gaps that required the development of a customized complex order display and a verification documentation workflow. Staff reported safety events were monitored for two years and compared to the year prior to go live.

Results: A workflow was enabled to capture real-time provider verification status during the time from ordering to the administration of chemotherapy. A customized display system was embedded in the EMR to provide a single screen view of the relevant parameters of chemotherapy doses including current and previous patient measurements of height and weight, dose adjustments, provider verifications, prior chemotherapy regimens, and a synopsis of the standard regimen for reference. Our system went live with 127 chemotherapy plans and has been expanded to 189 . Staff reported safety events decreased following implementation, particularly in the area of prescribing and transcribing by the second year of use.

Conclusions: We observed reduced staff reported safety events following implementation of CPOE for inpatient chemotherapy using an electronic verification workflow and an embedded custom clinical decision support page. This implementation demonstrates that CPOE can be safely used for inpatient chemotherapy, even in an extremely complex environment.

\section{Correspondence to:}

Daniel Martin

Seattle Cancer Care Alliance

825 Eastlake Ave.E.

Seattle, WA 98109

Tel.: (206) 2881284

Fax: (206) 7322187

Email: dmartin@seattlecca.org
Appl Clin Inform 2015; 6: 638-649

http://dx.doi.org/10.4338/ACl-2015-03-RA-0027

received: March 17, 2015

accepted in revised form: August 28, 2015

published: October 28, 2015

Citation: Martin DB, Kaemingk D, Frieze D, Hendrie P, Payne T. Safe implementation of computerized provider order entry for adult oncology. Appl Clin Inform 2015; 6: 638-649

http://dx.doi.org/10.4338/ACI-2015-03-RA-0027 


\section{Background}

Health IT is widely viewed as essential to reducing the cost of healthcare while improving quality, safety, and accessibility. The Institute of Medicine has called for automation, computerized reminders, and elimination of handwritten clinical data [1]. Use of computerized provider order entry (CPOE) systems for medication ordering is part of the meaningful use incentives [2]. CPOE provides legible, standardized, and complete orders, can reduce medication errors during various stages of the order management process [3-8]. However, CPOE also creates anticipated and unanticipated negative consequences, creating opportunities for medication errors that threaten patient safety $[9,10]$, including wrong patient orders [11] and selection of incorrect orders [12].

Oncology practice has lagged in CPOE adoption for a variety of reasons including cost, time pressures and aversion to change [13]. More importantly, institutions have been slow to embrace CPOE chemotherapy ordering because of safety concerns due to the complexity chemotherapy ordering. First, errors in chemotherapy administration can be lethal, thus national safety standards dictate that CPOE must include clear documentation of multiple provider verification [14]. Second, patient specific dosing schemes must be available in CPOE (e.g. body surface area (BSA)). Third, CPOE must allow chemotherapy to be precisely timed against other medications, creating complexity when drugs are held, rescheduled, or delayed. In chemotherapy ordering there are also significant potential advantages to CPOE. Electronic ordering can potentially provide maximum and cumulative dose alerts, decrease errors due to drug name confusion, and utilize clinical calculators with clear data sources.

General support for CPOE for oncology in the medical literature is only slowly developing and has been recently reviewed [15]. A few publications have attempted to quantify benefit with the implementation of CPOE for chemotherapy. A recent analysis showed a reduction of chemotherapy orders requiring rework from $12.6 \%$ to $2.2 \%$ after switching from preprinted order sets to CPOE [16]. CPOE in a large tertiary institution showed a reduction in prescribing errors from $9.5 \%$ using paper to $3.2 \%$ with CPOE for oral chemotherapy [17]. Chen and Lehman reported a 39\% reduction in medication-related patient safety events and a high level of provider acceptance using a commercially available CPOE system[18]. Theirs was the final unit at the center to adopt CPOE and required "considerable effort" to develop a customized implementation. Other studies have demonstrated similar results using various commercial and in-house developed systems [19-21]. A CPOE system was safely implemented at St. Jude Children's Research Hospital in 2010 after technical improvements addressed safety issues [22]. It required the creation of more than 3000 individual order sets, with the authors noting that "the resources required to safely implement CPOE for chemotherapy are extensive and require significant clinical experience to be most effective."

\subsection{Setting}

The SCCA is a National Comprehensive Cancer Network (NCCN) member institution and an NCI designated comprehensive cancer center operated jointly by the Fred Hutchinson Cancer Research Center, University of Washington, and Seattle Children's Hospital. Approximately 3000 unique patients are treated at the SCCA outpatient clinic annually and there are approximately 1300 hospital admissions for chemotherapy administration at the University of Washington Medical Center. The SCCA also performs 300 allogeneic and 200 autologous stem cell transplants annually. SCCA patients are often treated on research protocols and receive specialized care for uncommon or relapsed malignancies. Prior to $\mathrm{CPOE}$, inpatient chemotherapy was ordered on preprinted paper forms with doses approved by a pharmacy oversight committee. By policy, all non-standard chemotherapy requires two oncologist signatures.

\section{Objectives}

The objective of the work described in this paper was to safely deploy CPOE using a commercial system for inpatient chemotherapy administration in an academic teaching hospital and monitor safety events around this deployment. Our decision to go-live required the maintenance or improve- 
ment of existing safety standards for chemotherapy dosing using pharmacy approved chemotherapy forms prepopulated with standard drugs and doses. The project also demanded the development of a new and transparent dose validation workflow built around CPOE. A multidisciplinary pre-implementation analysis yielded significant but surmountable challenges that required customization to resolve and provide a much safer and transparent system. The purpose of this paper is to describe our customization efforts so that these tools can be shared or recreated in other EMRs to enhance the safety of electronic ordering of chemotherapy.

\section{Methods}

\subsection{CPOE Feasibility and Planning at the University Washington}

In 2010, the SCCA began planning transition from paper to electronic chemotherapy ordering as part of the development of inpatient CPOE in conjunction with the institutional rollout scheduled for May 2012. Support for the effort and a background for establishing safety systems was provided through the successful implementation of CPOE at St. Jude's in 2011, using an EMR suite from the same vendor. To determine the feasibility of CPOE for chemotherapy, a multidisciplinary team including physicians, pharmacists, nurses, and IT specialists was established. The committee established five principles for safe chemotherapy administration using CPOE: 1) chemotherapy orders must be standardized, adhering to the concept of standard regimens established in preprinted paper orders, 2) all components of an electronic chemotherapy order must be visible to all users of the EMR, i.e. displaying critical data including biometrics, order details, and verification details; 3 ) orders must contain embedded reference text providing the standard of care for each regimen; 4) chemotherapy order sets must be maintainable over long periods of time; and 5) the process of executing orders must be easy to learn so that persons involved in the administration of chemotherapy can be rapidly and effectively educated for safe administration.

\subsection{Identification of Gaps in CPOE for Chemotherapy}

The CPOE chemotherapy development committee selected the rituximab-hyper CVAD leukemia regimen (R-hyper CVAD) [23] as a test regimen because of its complexity, including multiple drugs, delivery methods, days of therapy and supportive care that is precisely timed. A test domain was built and a chemotherapy order set (called a PowerPlan) was developed for R-hyper CVAD. Some advantages of CPOE were obvious. Reference text could be embedded in the orders with hyperlinks to relevant literature or practice standards. Orders were clear. Doses and units of dosing (e.g. mg/kg) could be prepopulated as could parameters such as height, weight and creatinine. Orders placed using CPOE were electronically routed to pharmacy without any need for transcription. However, a number of critical workflow differences between chemotherapy CPOE and CPOE for other services became apparent that had profound influence on the process; accommodating these differences was key to our successful implementation. The first challenge was ensuring that critical ordering parameters (e.g. height and weight) entered by the prescriber using the dose calculator were later clearly available to pharmacists and nurses for order verification. A second critical challenge was developing embedded EMR tools and workflows to document and display the sequential provider, pharmacist and two nurse verifications prior to mixing and administering chemotherapy. For prescribing non-standard chemotherapy (e.g. salvage, research, and unpublished regimens), our institution requires a second physician verification, which had to be captured during CPOE.

\subsection{Capture and Display of Order Details}

The capture and display of order details posed the greatest challenge in using CPOE for chemotherapy. With electronic ordering, each drug requiring weight-, BSA-, or creatinine-based dosing must be individually dosed using an electronic dose calculator ( Figure 1 ). The dose calculator is defaulted to the standard dose including units, with a prespecified rounding rule. However, for every drug in a multi-drug regimen, the physician can potentially change any of the patient parameters, 
standard drug doses, or preselected units (e.g. $\mathrm{mg} / \mathrm{m}^{2}$ ) that prepopulate the dose calculator. Consequently, there is potential for multiple weights or heights to be used within a single multi-drug chemotherapy order. This is in contrast to a paper-based chemotherapy order where the physician typically provides an easily viewed single height, weight, and creatinine to be used for all dose calculations. Likewise, adjustment of standard drug doses on a paper chemotherapy order (with drugs, doses, and units pre-printed) is highly visible with adjacent text clarifying the rationale. In summary, CPOE for chemotherapy has the potential to allow multiple points of adjustment in every electronic order in a plan which must be individually double checked by verifying providers.

In the Cerner Millennium EMR, viewing of order details for any medication ordered using the dose calculator requires navigation through three hierarchical levels. This process obscures reference text and historical patient data (height, weight, etc.). Back and forth navigation between order details and pertinent information is challenging, and side-by-side presentation infeasible in the native EMR. We felt this to be a significant step backward from a preprinted form where a single sheet of paper provided all ordering details. We regarded this as a potential safety hazard as it would require printing multiple screens from the EMR to verify numerous details using side-by-side comparison of paper to electronically displayed information. Thus, for CPOE for chemotherapy to proceed safely, we needed to devise a solution to provide transparency of chemotherapy dosing for verification. This situation led to the development of an embedded webpage (called an Mpage) within the EMR that allowed single panel viewing of all the pertinent information of the order, recapitulating, and improving upon the paper process.

\subsection{Development of a Chemotherapy Dosing Summary Page}

We developed a custom page embedded within the EMR to display details from chemotherapy order sets (PowerPlan). The Mpage utilizes HTML formatting and Java Script to extract all relevant details used in dosing each chemotherapy drug from the data entered in the EMR using dose calculator. These details are displayed in a single screen in a fashion resembling a paper chemotherapy order sheet, eliminating the need to toggle between EMR locations. The Mpage can apply logical comparisons to extracted data and used formatting changes (e.g. highlighting) to emphasize particular fields, but does not have the ability to write back to the EMR data tables.

\section{Results}

\subsection{Chemotherapy Dosing Summary Page}

The top section of the chemotherapy dosing summary Mpage ( $\triangleright$ Figure 2 ) displays a chronological table of weights and heights where the user can easily identify anomalous values and trends (such as those in Figure 2 dated 10/30/13). Below this the Mpage shows the standard regimen doses extracted from the chemotherapy PowerPlan. The ordering provider and cycle number are identified. A table shows the verifications performed including dates and time of completion and provider comments. The status of the verification (e.g. complete, not reviewed) is displayed and chemotherapy is not given unless the proper verifications are ordered (discussed below). A chemotherapy table under the verifications includes rows for each drug and columns for the standard dose and units of dosing immediately adjacent to the prescribed doses (including units) and other extracted order details. Dose reduction and prescribed dose are specifically called out because these are both potential methods of reducing the dose given to the patient. The ordering provider can manually reduce the prescribed dose (e.g. $750 \mathrm{mg} / \mathrm{m}^{2}$ for cyclophosphamide), or use the dose adjustment in the dose calculator to reduce the dose calculated by a percent (as done in $>$ Figure 2 for cyclophosphamide). The column view (basic or expanded) can be adjusted by the user. The Mpage also displays previously administered PowerPlans allowing providers additional reference data during verification. This includes the dates of prior therapies and any previous dose adjustments for toxicities.

The Mpage was felt to be far superior to the manual checking required using the functions provided in the native EMR. Failure modes were tested through exhaustive scripting to establish 
scenarios where incorrect, misleading, or outdated information could be provided resulting in an error. It has been in use since the system went live in August of 2012 and has been iteratively modified to improve clarity. The Mpage is capable of logical comparisons between fields. Development plans include comparison between the "Regimen Dose" and "Prescribed Dose" to highlight any potential dose adjustments or accidental adjustments of dosing units.

\subsection{Capture and Display of Verifications}

The SCCA follows national guidelines for safe administration of chemotherapy and requires that signed chemotherapy orders are verified by a pharmacist followed by two nurses prior to administration [14]. At UW, CPOE implementation necessitated the development of a verification tool with clarity of authorship and time of verification. This goal was achieved by inserting electronic verification orders in all chemotherapy PowerPlans within a separate phase ( Figure 3 ). The order is defaulted to "NOT REVIEWED", with the exception of the second attending verification order which is defaulted to "Not Required". In practice, the provider signing the chemotherapy PowerPlan is the person ordering the chemotherapy (and the verifications). The verification orders, marked "verification required" at the time of ordering are then modified to "VERIFICATION COMPLETE" by the verifying providers. The verification order allows one to add clarifying references or comments. All changes of verification orders and comments propagate onto the chemotherapy dosing summary Mpage upon signature, providing visibility of all safety checks in one location with a clear date and time stamp. Incomplete verifications display in red and completed verifications in black ( Figure 2). We have found that the ability to place comments in the verifications orders provides clarity to verifying providers, reduces phone calls, and administration delays.

The verification orders also allow for attending verification when the orders are written by a nonattending provider. At UW, non-attending providers (e.g. oncology fellows, PA's and ARNP's, but not residents) can order chemotherapy which must be cosigned by an attending before administration. The verification orders in the chemotherapy PowerPlan are the mechanism for this. Non-standard chemotherapy ordered on paper orders required two attendings signatures by policy. When ordering, the ordering provider adjusts the second attending verification order to "REVIEW REQUIRED" and the second attending subsequently modifies this order to verification complete. Consideration was given to defaulting this field to "REVIEW REQUIRED" rather than "Not required". However the fraction of orders requiring a second attending signature is in the range of $2 \%$, meaning this field would be adjusted for nearly every chemotherapy order. Forgetting to change this setting was thought to be a real possibility that would result in disrupted workflow and patient delays. Given these considerations it was decided to default the second attending review to "Not required".

\subsection{Clinical Reception}

Reviews of the system were positive based on informal reviews with staff work groups. The chemotherapy dosing page was incorporated into the standard work of clinical pharmacists and verifying nurses. These provider groups found that the side-by-side comparison of the standard doses in the synopsis with the doses ordered was facile and maintained their confidence and comfort with their workflow compared with the paper based system to which they were accustomed. The display of dose reductions and a standard location to find the rationale was also regarded as safety improvement and reduced delays to obtain clarification. Both nursing and pharmacy leadership reported satisfaction with the improved clarity and reduced lag time of electronic orders compared to the paper ordering. Physicians have benefitted from the ability to enter, sign or verify orders remotely without the requirement of transporting the paper document to the pharmacist and subsequently to the hospital. The orders can be viewed by any provider, pharmacist or nurse simultaneously at any time. Finally, CPOE has improved the ability to recognize and resolve issues of absent, incomplete, or incorrect orders. 


\subsection{Scale of Use}

CPOE for inpatients has been used inpatient care since August 2012. CPOE went live with 127 chemotherapy PowerPlans built and there are currently 189 available. This number has stabilized. Decisions regarding the maintenance or adjustment of our library are assigned to our Order Set Governance committee, which meets twice monthly. The scope of the PowerPlans includes all subspecialties of hematology/oncology including but not limited to malignant hematology, sarcoma, neuroncology, gyn-oncology, and blood and marrow transplantation. In the first two years of ordering electronically, there were approximately 1300 chemotherapy PowerPlans ordered annually on the inpatient service, including intrathecal and intra-hepatic chemotherapy. After go live, CPOE became the only permissible method to order chemotherapy with the exception of clinical trials involving investigational drugs (which are not available in the electronic ordering catalog). Orders for investigational drugs represented roughly 3\% of all chemotherapy orders. Chemotherapy regimens for which no power plan exists are ordered using CPOE using a generic chemotherapy template that allows the selection of standard orders for hydration, antiemetics, growth factors, and a verification phase. Over $98 \%$ of all orders after go live have been ordered using CPOE.

\subsection{Safety Events}

Potential or actual safety events at UWMC are reported via Patient Safety Net (PSN). As part of the reporting mechanism, these events are categorized according to the origin and severity of the event ( Table 1). Typical examples of each category are given. Administering events include examples of incorrect infusion rate, infusion of only partial dose, delays, or incorrect timing of medications with a premedication. Dispensing events include those with incorrect tubing or bag size, an incorrect diluent, leaking bags, or mislabeling. Monitoring events include events in which desired urinary $\mathrm{pH}$ was not achieved prior to chemotherapy infusions, mistimed drug levels, or inappropriate timing of rescue medication (i.e. leucovorin or mesna). Prescribing/ordering events include incorrect dose, incorrect weight used, or lack of necessary supportive care, use of incorrect PowerPlans or lack of documentation on deviation from standard therapy. Transcribing/ordering processing events include delays from orders needing validation, subsequent dose mistiming after an initial delay, delays due to waiting for pharmacokinetic results, and requests for non-standard dose times. In the first year post CPOE implementation, there was a small numeric increase in total events reported as compared to the previous year ( 90 vs. 86). This rise may have been due to an increase in such events or because hospital staff were encouraged to report potential safety concerns.

There was a noticeable uptick in the first year of CPOE in prescribing/ordering events and the type of events seen changed. The reasons for this uptick are multiple, and include unfamiliarity with the system causing errors of omission and incorrect use of the system. After CPOE went live, there were no episodes of unreadable orders, "trailing zero" doses, or orders for which the BSA was miscalculated (all reported prior to CPOE). There were a variety of reported events that included issues due to unfamiliarity: signed orders left unverified by the attending causing delays, orders placed on the wrong encounter, use of a powerplan for a regimen including an investigational drug (remains on paper) and failure to document reasons for dose reductions for verifying staff. Incorrect ordering events also included the inappropriate modification of standard plans through the addition of chemotherapy medications, creating a discrepancy between the ordered chemotherapy and the standard dose information. Dosing events were also reported such as orders using actual rather than adjusted ideal body weight, or wrong route, (e.g. intravenous instead of subcutaneous administration of bortezomib).

In the second year post CPOE implementation, the number of total reported events dropped by approximately $20 \%$ as compared to the year prior to implementation. The number of events which led to additional treatment or caused temporary harm was not affected by the implementation (five in the year prior to CPOE and five and four the following two years). These data are consistent with the expectation that CPOE reduces errors with the use of pre-built order sets and by minimizing the need for pharmacy transcription. It also demonstrates that electronic provider order entry of high risk medications may safely be done with careful consideration of the design process. 


\section{Discussion}

Chemotherapy ordering using CPOE was successfully implemented for the inpatient service at the University of Washington in 2012 and has been operational without interruption or sentinel safety event for two years. PSN data showed a similar number of total events in the first year post implementation and a decreased number of total events in the second year of use of CPOE. This implementation required the development of a number of tools to address safety and workflow requirements and which allowed continued compliance with the guidelines adopted by the institution for chemotherapy. Our EMR vendor's design in combination with our oncology Mpage allowed us to develop a process that avoids the high importance failure modes in chemotherapy administration previously identified (wrong patient, wrong dose, wrong medication, and miscalculation) [24]. This is achieved through ordering within the patient's EMR, using a limited menu of options, populating data from the EMR into a dose calculator, and performing and clearly documenting multi-person verifications. We based our workflow on the critical parameters described for pediatric chemotherapy administration [18] in that we provide clarity of the prescriber's intention and independent checks to allow recovery from human error. This manuscript describes an inpatient oncology program with a limited set of chemotherapy PowerPlans. The vast majority of chemotherapy administered at the SCCA is done so in the outpatient setting, explaining the large difference in power plan numbers between the effort at St. Judes and our own. The number of PowerPlans for our outpatient CPOE effort is roughly five times as large because of an increased number of standard regimens and a large number of clinical trials.

\subsection{Limitations}

Our findings have limitations. This was not a controlled trial so it is not possible to attribute rise or fall in recorded safety events to the interventions we describe. It is also possible that the true adverse event rate was different than shown in our reporting system data, since incident reporting systems may not detect true adverse drug event rates [25]. Staff reported safety data could be biased in a number of ways because of efforts to encourage reporting around the period of CPOE go live and because staff workload and time logged into a computer may have changed at that time providing more or less time to file reports. Because of the lack of formalized data gathering, the possibility of bias, and the relatively small number of events, we have not conducted a statistical analysis. Data was gathered at a single academic center with highly standardized procedures and may not be generalizable to all environments. The acceptance of the system by staff and providers was not measured using a formal survey tool. The order catalog includes 78 clinical trials. Clinical trials involving investigational drugs continue to use paper ordersets. In this case an inpatient pharmacist transcribes the medication orders into the EMR through the pharmacy EMR module. Finally, the Mpage does not write back to the data tables of the native EMR. Logical comparison between fields allows highlighting that can call out potential errors or discrepancies to verifying staff. This does not fire alerts with in the native EMR at the time of ordering or elsewhere in the order administration process.

\section{Conclusions}

CPOE for chemotherapy has been successfully and safely implemented in a complex inpatient oncology and transplant service. Planning efforts identified safety gaps in mechanisms for communicating the details in for chemotherapy dosing to providers which were overcome by developing an embedded chemotherapy dosing summary page which satisfied nursing and pharmacists. A strategy was also developed for documenting and displaying provider verifications within the EMR. With this system in place, CPOE has been used for ordering chemotherapy for over two years without a large variation documented safety events. 


\section{Clinical Relevance Statement}

We demonstrate that CPOE using an electronic verification workflow and an embedded custom clinical decision support page can be safely used for inpatient chemotherapy, and was correlated with reduced staff reported safety events.

\section{Conflict of Interest}

None of the authors have any relationships with organizations or industry regarding the contents of this manuscript that could be viewed as potential conflicts of interest.

Protection of Human and Animal Subjects

Not applicable. 


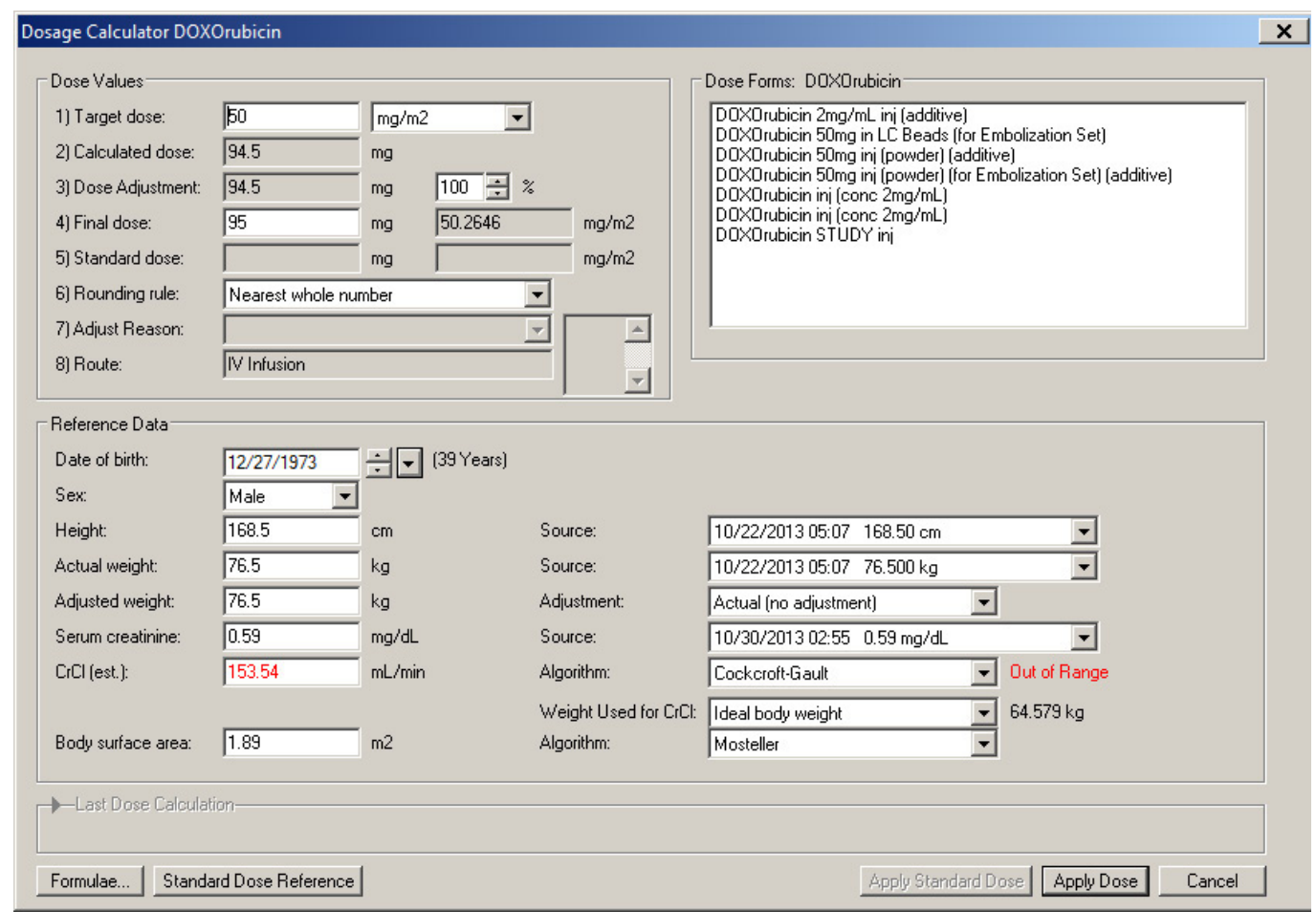

Fig. 1 Dosage Calculator. The parameters in the dosage claculator are auto-populated, but each parameter can be manually overwritten for each drug. The calculator provides a mechanism to adjust doses (3) and to provide an adjustment reason (7) using either standard text or a free text box.

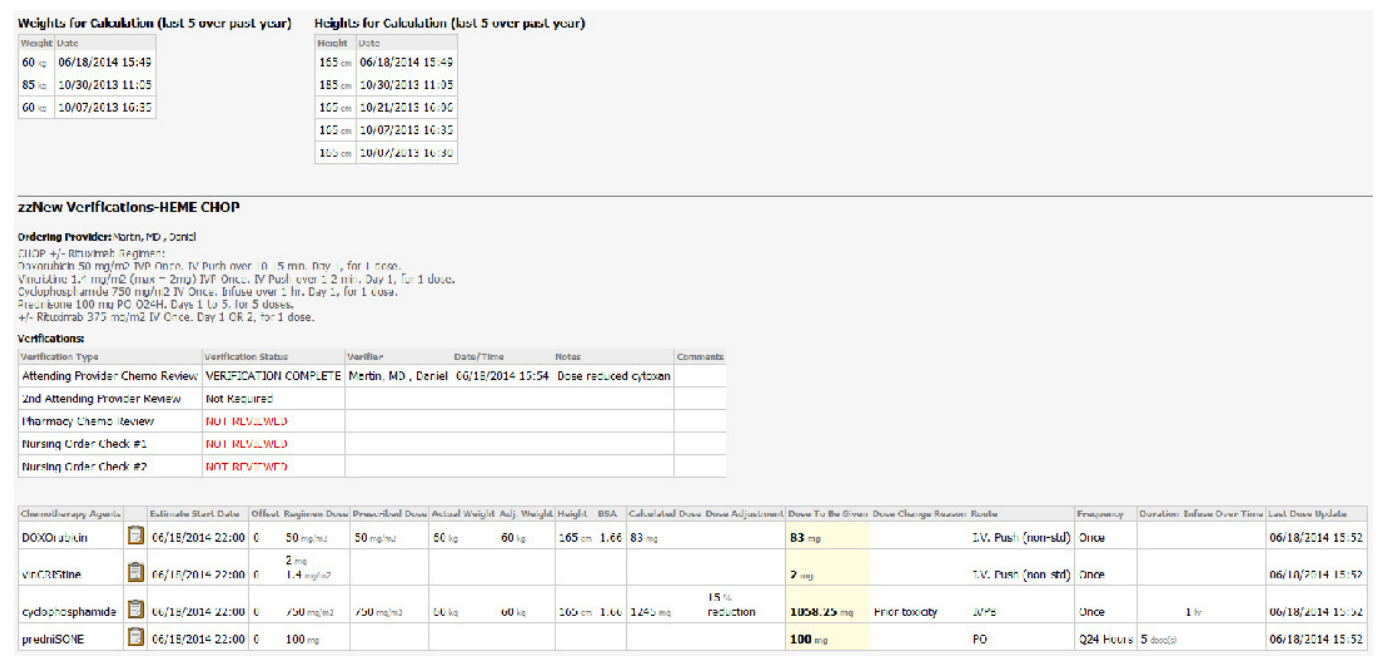

Fig. 2 Chemotherapy Dosing Summary Mpage. Tables above display the most recent five height and weight values. A synopsis of the standard chemotherapy regimen including doses and details is included for references. Verifications are shown and colored according to status including provider name and comments. The actual doses ordered and the data used to make the calculations is displayed in a tabular format with the reference doses included for comparison.

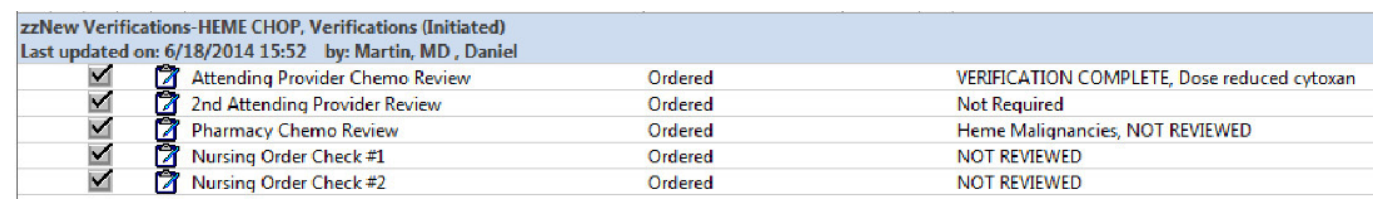

Fig. 3 Electronic Verification Order Phase in a Chemotherapy PowerPlan. 
Table 1 Origin of Patient Safety Net Events for one Year Prior to CPOE Implementation and Two Years Post CPOE Implementation

\begin{tabular}{|l|l|l|l|}
\hline & 1 Year Pre-CPOE & 1st Year CPOE & 2nd Year CPOE \\
\hline Administering & 30 & 24 & 25 \\
\hline Dispensing & 12 & 14 & 10 \\
\hline Monitoring & 16 & 15 & 14 \\
\hline Prescribing/ordering & 10 & 20 & 7 \\
\hline Transcribing/order processing & 18 & 17 & 11 \\
\hline Grand Total & 86 & 90 & 67 \\
\hline
\end{tabular}




\section{References}

1. Kohn LT, Corrigan JM, Donaldson MS [editors]. To Err is Human. Building a safer health system. Institute of Medicine; Washington (DC): National Academies Press (US) 2000.

2. Blumenthal D, Tavenner M. The „meaningful use" regulation for electronic health records. N Engl J Med, 2010; 363(6): 501-504.

3. Ammenwerth E, Schnell-Inderst P, Machan C, Siebert U. The effect of electronic prescribing on medication errors and adverse drug events: a systematic review. J Am Med Inform Assoc 2008; 15(5): 585-600.

4. Bates DW, Leape LL, Cullen DJ, Laird N, Petersen LA, Teich JM, Burdick E, Hickey M, Kleefield S, Shea B, Vander Vliet M, Seger DL. Effect of computerized physician order entry and a team intervention on prevention of serious medication errors. JAMA 1998; 280(15): 1311-1316.

5. Bates DW, Teich JM, Lee J, Seger D, Kuperman GJ, Ma'Luf N, Boyle D, Leape L. The impact of computerized physician order entry on medication error prevention. J Am Med Inform Assoc 1999; 6(4): 313-321.

6. Devine EB, Hansen RN, Wilson-Norton JL, Lawless NM, Fisk AW, Blough DK, Martin DP, Sullivan SD. The impact of computerized provider order entry on medication errors in a multispecialty group practice. J Am Med Inform Assoc 2010; 17(1): 78-84.

7. Kaushal R, Kern LM, Barron Y, Quaresimo J, Abramson EL. Electronic prescribing improves medication safety in community-based office practices. J Gen Intern Med 2010; 25(6): 530-536.

8. Reckmann MH, Westbrook JI, Koh Y, Lo C, Day RO. Does computerized provider order entry reduce prescribing errors for hospital inpatients? A systematic review. J Am Med Inform Assoc 2009; 16(5): 613-623.

9. Ash JS, Sittig DF, Poon EG, Guappone K, Campbell E, Dykstra RH. The extent and importance of unintended consequences related to computerized provider order entry. J Am Med Inform Assoc 2007; 14(4): 415-423.

10. Koppel R, Metlay JP, Cohen A, Abaluck B, Localio AR, Kimmel SE, Strom BL. Role of computerized physician order entry systems in facilitating medication errors. JAMA 2005; 293(10): 1197-1203.

11. Adelman JS, Kalkut GE, Schechter CB, Weiss JM, Berger MA, Reissman SH, Cohen HW, Lorenzen SJ, Burack DA, Southern WN. Understanding and preventing wrong-patient electronic orders: a randomized controlled trial. J Am Med Inform Assoc 2013; 20(2): 305-310.

12. Nanji KC, Rothschild JM, Salzber C, Keohane CA, Zigmont K, Devita J, Gandhi TK, Dalal AK, Bates DW, Poon EG. Errors associated with outpatient computerized prescribing systems. J Am Med Inform Assoc 2011; 18(6): 767-773.

13. Warner J, Hochberg E. Where is the EHR in oncology? J Natl Compr Canc Netw 2012; 10(5): 584-588.

14. Neuss MN, Polovich M, McNiff K, Esper P, Gilmore TR, LeFebvre KB, Schulmeister L, Jacobson JO. 2013 updated American Society of Clinical Oncology/Oncology Nursing Society chemotherapy administration safety standards including standards for the safe administration and management of oral chemotherapy. Oncol Nurs Forum 2013; 40(3): 225-233.

15. Bubalo J, Warden BA, Wiegel JJ, Nishida T, Handel E, Svoboda LM, Nguyen L, Edillo PN. Does applying technology throughout the medication use process improve patient safety with antineoplastics? J Oncol Pharm Pract 2013; 20(6): 445-460.

16. Meisenberg BR, Wright RR, Brady-Copertino CJ. Reduction in chemotherapy order errors with computerized physician order entry. J Oncol Pract 2014; 10(1): e5-e9.

17. Collins CM, Elsaid KA. Using an enhanced oral chemotherapy computerized provider order entry system to reduce prescribing errors and improve safety. Int J Qual Health Care 2011; 23(1): 36-43.

18. Chen AR, Lehmann CU. Computerized provider order entry in pediatric oncology: design, implementation, and outcomes. J Oncol Pract 2011; 7(4): 218-222.

19. Cho E, Kim HJ, Kim GM, Kum J, Chung HK, Lyu CJ, Ahn JB, Shin SJ. Assessment of efficiency and safety of the comprehensive Chemotherapy Assistance Program for ordering oncology medications. Int J Med Inform 2013; 82(6): 504-513.

20.Huertas Fernandez MJ, Baena-Canada JM, Martinez Bautista MJ, Arriola Arellano E, Garcia Palacios MV. Impact of computerised chemotherapy prescriptions on the prevention of medication errors. Clin Transl Oncol 2006; 8(11): 821-825.

21. Voeffray M, Pannatier A, Stupp R, Fucina N, Leyvraz S, Wasserfallen JB. Effect of computerisation on the quality and safety of chemotherapy prescription. Qual Saf Health Care 2006; 15(6): 418-421.

22. Hoffman JM, Baker DK, Howard SC, Laver JH, Shenep JL. Safe and successful implementation of CPOE for chemotherapy at a en's cancer center. J Natl Compr Canc Netw 2011; 9 (Suppl 3): S36-S50.

23. Kantarjian HM, O’Brien S, Smith TL, Cortes J, Giles FJ, Beran M, Pierce S, Huh Y, Andreeff M, Koller C, Ha CS, Keating MJ, Murphy S, Freireich EJ. Results of treatment with hyper-CVAD, a dose-intensive regimen, in adult acute lymphocytic leukemia. J Clin Oncol 2000; 18(3): 547-561. 
24. Kim GR, Chen AR, Arceci RJ, Mitchell SH, Kokoszka KM, Daniel D, Lehmann CU. Error reduction in pediatric chemotherapy: computerized order entry and failure modes and effects analysis. Arch Pediatr Adolesc Med 2006; 160(5): 495-498.

25. Cullen DJ, Bates DW, Small SD, Cooper JB, Nemeskal AR, Leape LL. The incident reporting system does not detect adverse drug events: a problem for quality improvement. Jt Comm J Qual Improv 1995; 21(10): 541-548. 